\title{
A systems approach to the management of acute surgical pain and reduction of opioid use: the approach of Oakville Trafalgar Memorial Hospital
}

\section{Duncan Rozario, MD}

Accepted Feb. 14, 2020

\section{Correspondence to:}

\section{Rozario}

Oakville Trafalgar Memorial Hospital 3001 Hospital Gate

Oakville, ON L6M OL8

drozario@haltonhealthcare.com

DOI: $10.1503 /$ cjs.000719

\section{SUMMARY}

Surgeons are among the highest prescribers of opioids, accounting for $56 \%$ of new starts of opioids in Ontario in 2016. ${ }^{1}$ At times we may not have an appropriate systems-based approach to the management of acute pain, which may lead to suboptimal management or unintended long-term dependency issues. We present the newly developed integrated systems approach of our Department of Surgery (www.oakvillesurgery.com) to pain management in the setting of acute surgical pain and show how surgeons can make a substantial impact in this important aspect of care.

akville Trafalgar Memorial Hospital is a 469-bed facility in Oakville, Ontario; 13717 surgical procedures were performed at the hospital in 2017-18.

\section{ACUTE PAIN OVERVIEW}

Surgeons, while accounting for the majority of opioid starts, may not appreciate how an integrated approach to pain management can result in a lower requirement for opioids, yet better functional status. Canada has the second highest per capita use of opioids in the world, ${ }^{1}$ and health care providers have a responsibility to use the best evidence-based approach to maximize pain management and minimize unintended long-term consequences such as dependency, chronic pain, increased costs and the diversion of narcotics. We should attempt to use opioid-reduction strategies and consider eliminating the use of opioids in selected cases, as it has been shown that the combination of acetaminophen and nonsteroidal antiinflammatory drugs (NSAIDS) can reduce opioid usage by more than $50 \%$ and at times eliminate the need for opioids altogether. ${ }^{2}$ After starting our widespread patient experience surveying of our day surgery patients, ${ }^{3}$ one of the recurrent issues we identified was inconsistent postoperative pain management. Our Acute Pain Service, under the Department of Anesthesia and the Department of Surgery, has developed an integrated pain system for our day surgery patients, divided into 3 approaches: preoperative, intraoperative and postoperative.

\section{Preoperative APPROACH}

Patient education is a vital component of pain management, and we have developed a patient guide to pain management after surgery that outlines our recommendations and explains pre-emptive analgesia, use of multimodal postoperative pain control and alternative supportive therapies. We also attempt to manage expectations, explaining that patients will not be pain-free after surgery, but will have adequate pain control to maintain their functional status (i.e., be able to move and conduct their activities of 
daily living with adequate comfort). This information is provided in written form and reinforced verbally throughout the patient's journey in the surgical program.

Pre-emptive analgesia involves administering pain medication before surgery to reduce or eliminate postoperative pain and to reduce or eliminate opioid requirements. Our anesthesiologists order these medications for our surgical patients before surgery. To be consistent, we are developing a medical directive: a Medical Algorithm for Preoperative Pain Management. This directive ensures that all patients in whom there are no medical contraindications receive the following preoperatively: acetaminophen $1 \mathrm{~g}$ and celecoxib $200 \mathrm{mg}-400 \mathrm{mg}$ for all procedures, and gabapentin (dose adjusted $100 \mathrm{mg}-$ $400 \mathrm{mg}$ based on age) for intermediate and major elective procedures. We have reviewed contraindications such as hepatic dysfunction with the use of acetaminophen and renal dysfunction and peptic ulceration with NSAIDS. The importance of preoperative administration cannot be emphasized enough, as the NSAID-mediated blockage of cycloxygenase-mediated prostaglandin production, which leads to inflammation and pain, is drastically more effective before the surgical insult.

\section{INTRAOPERATIVE APPROACH}

Bupivacaine is an amino-amide long-acting local anesthetic that can provide up to 15 hours of sensory blockage. In addition, bupivacaine has been shown to be bacteriostatic at clinical doses. At a dose of $2 \mathrm{mg} / \mathrm{kg}$, the recommended concentration of $0.25 \%(2.5 \mathrm{mg} / \mathrm{mL})$ for sensory blockage allows the instillation of up to $56 \mathrm{cc}$ of $0.25 \%$ bupivacaine for pre- or postoperative incisional instillation in a $70 \mathrm{~kg}$ adult (https://www.pfizer.ca/pm/ en/MARCAINE.pdf). Preoperative installation is likely superior. Bupivacaine has been shown to reduce postoperative opioid use and to have anti-inflammatory activity. We are instituting an educational campaign to ensure that all surgical staff utilize this medication appropriately for all indicated cases with cutaneous incisions. Alternative agents include levobupivacaine and ropivacaine. Liposomal bupivacaine, which has a duration of action of several days, is a new formulation that may have substantial clinical value. Surgical staff also support the anesthesiologist providing neuroaxial and regional blocks given their proven value in optimizing pain management and opioid reduction.

\section{Postoperative APPROACH}

Dr. Feinberg and colleagues ${ }^{4}$ at North York General Hospital showed that the majority of patients use only a fraction of prescribed narcotics after surgery, and we instituted a multimodal, minimal opioid, day surgery prescription that consists of acetaminophen $1 \mathrm{~g}$ every
6 hours for 48 hours, ibuprophen $400 \mathrm{mg}$ every 6 hours for 48 hours, and hydromorphone $1-2 \mathrm{mg}$ every 6 hours as needed (maximum 10 tablets to fill only if needed) if there are no medical contraindications. After the stated time period, the over-the-counter medications are used on an as-needed basis. We include an instruction sheet with our prescription so patients have a clear understanding of our recommended approach to pain management (Appendix 1, available at canjsurg. ca/000719-a1).

The author's prescribing pattern of 30 tablets of $1 \mathrm{mg}$ hydromorphone alone to all day surgery patients changed in April 2018 to follow the prescription above. Over the 6-month period of January to June 2019 for 105 consecutive day-surgery cases (cholecystectomy, hernia, breast surgery), $79 \%$ of patients used no postoperative narcotics, and the remaining $21 \%$ used 84 tablets in total. Only 2 patients required a refill of their narcotic prescription.

Clear guidelines regarding the number of opioid tablets that should be prescribed exist for specific procedures. ${ }^{5}$ We have substantially reduced our opioid prescribing in our day surgery population. An important aspect of a low narcotic protocol is an early assessment of response to therapy. We are developing a postoperative program based on feedback from our surgical day care survey to improve patient satisfaction, where the attending surgeon calls or has a virtual video visit with their patient. We are using the Reacts platform (an integrated, collaborative tool for health care professionals; www. reacts.com). This allows an assessment of functional pain status and a determination whether an additional opioid prescription is needed, in a minority of cases. This call also allows us to more clearly communicate patients' operative findings and improve their experience of care.

\section{Conclusion}

Increasingly, the management of postoperative pain is moving away from a sole dependency on opioids and resulting in equivalent pain management. Clear guidelines exist for the optimal management of postoperative pain for specific procedures (PROSPECT [procedurespecific postoperative pain management]; https:// esraeurope.org/prospect/). A recent study looking at outpatient surgery found that only $45 \%$ of patients filled their opioid prescription after laparoscopic cholecystectomy or open hernia repair when presented with a robust multimodal prescription; $33 \%$ used no opioids, and the median opioid consumption was only 5 pills (oral morphine equivalent of 25). ${ }^{6}$ Optimal pain control improves rehabilitation after surgery, reduces chronic pain, and enhances return to work and daily activities - a clear economic benefit to society. We must continue to use an evidence-based approach to optimize 
postoperative pain control and ensure robust guidelines are developed based on science. In January 2020, the World Health Organization retracted its guidelines for opioid use after undue commercial influence was identified (https://www.ncbi.nlm.nih.gov/pmc/articles/ PMC6933427/).

Given the epidemic of inappropriate opioid use in North America, caregivers have a duty to use these medications with appropriate indications. In some cases, prescription opioids may serve as a gateway drug to dependency or the abuse of other agents. A robust systems-based approach that includes patient education and a multimodal approach through the surgical journey, including an assessment after surgery, has the potential to provide better care and reduce opioid utilization at the same time. In addition, we are exploring alternative modalities, such as guided imagery meditation, for our patients to improve their experience after surgery. Our colleagues in anesthesiology have extensive experience in multimodal pain control; a collaborative approach will lead to better collegiality and better patient care and experiences.

Acknowledgement: The author thanks Dr. Joseph Kay, retired chief of anesthesiology, for his leadership and guidance to the surgical program as the Acute Pain Service was developed; Dr. Kay graciously and patiently taught many surgeons the art and science of pain control.

Affiliation: From the Oakville Trafalgar Memorial Hospital, Oakville, Ont.

Competing interests: None declared.

\section{References}

1. Report HQOS. Starting on opioids. Available: www.hqontario.ca/ System-Performance/Specialized-Reports/Starting-on-Opioids-in -Ontario (accessed 2020 Feb. 14).

2. Martinez L, Ekman E, Nakhla N. Perioperative opioid-sparing strategies: utility of conventional NSAIDs in adults. Clin Ther 2019; 41:2612-28.

3. Rozario D. How well do we do what we do and how do we know it? The importance of patient reported experience measures in assessing our patient's experience of care. Can 7 Surg 2019;62:1-3.

4. Feinberg AE, Chesney TR, Srikandarahah S, et al. Opioid use after discharge in postoperative patients - a systematic review. Ann Surg 2018;267:1056-62.

5. Overton HN, Hanna MN, Bruhn WE, et al. Opioid-prescribing guidelines for common surgical procedures: an expert panel consensus. 7 Am Coll Surg 2018;227:411-18.

6. Hartford LB, Van Koughnett JAM, Murphy PB, et al. Standardization of outpatient procedure (STOP) narcotics: a prospective non-inferiority study to reduce opioid use in outpatient general surgical procedures. 7 Am Coll Surg 2019;228:81-8. 